\title{
CONCEPÇÃO DE INTERFACES PARA WEBSITES DE BIBLIOTECAS UNIVERSITÁRIAS: PROJETO, ELABORAÇÃO E GESTÃO DE INFORMAÇÃO EM MEIO DIGITAL
}

INTERFACES DESIGN FOR UNIVERSITY LIBRARIES WEBSITES: DESIGN, DEVELOPMENT AND MANAGEMENT INFORMATION IN DIGITAL MEDIA

Alexandre Ribas Semeler ${ }^{1}$

\begin{abstract}
Resumo:Aborda o desenvolvimento e a implantação de websites para Bibliotecas Universitárias. Tem como objetivo investigar as ferramentas, as tecnologias e as metodologias necessárias para o projeto e a concepção de interfaces de websites. Discute o fluxo de trabalho e o desenvolvimento de websites, concentrando esforços no projeto, na elaboração e na gestão da informação em meio digital. Aponta aspectos necessários para o trabalho com projetos de design de navegação, identidade visual e implementação de sistemas de gerenciamento de conteúdo.
\end{abstract}

Palavras-chave: Websites para Bibliotecas Universitárias. Metodologia de Desenvolvimento Web. Design de navegação. Sistemas de Gerenciamento de Conteúdo. Mashup. Webometria.

Abstract: Discusses the development and implementation of university libraries websites. Aims to investigate the required tools, technologies and methodologies for websites interface design. Discusses the workflow and development of websites focusing on design, development and management of information in digital media. Points out the necessary aspects for working with navigation design projects, visual identify and implementation of content management systems.

Keywords: University libraries websites. Web development methodology. Navigation design. Content Management Systems. Mashups. Webometrics.

\section{INTRODUÇÃO}

O desenvolvimento das tecnologias digitais possibilita a criação de novas práticas bibliotecárias ligadas ao tratamento e à organização de informações digitais

\footnotetext{
${ }^{1}$ Mestre em Comunicação e Informação pela Universidade Federal do Rio Grande do Sul (UFRGS). Bibliotecário Documentalista do Instituto de Geociências da UFRGS; Porto Alegre, RS, Brasil. E-mail: alexandre.semeler@ufrgs.br

Enviado em: 26/07/2011 - Aceito em: 30/10/2012.
} 
na web. Nesse contexto, o trabalho com websites revela-se como uma das novas áreas de interesse para os profissionais da informação.

A prática com websites envolve uma série de questões ligadas à informação digital. Ela articula questões relacionadas às novas tecnologias, aos estudos de usuários, ao design da informação e à gestão da informação em meio digital. Assim, este relato de experiência se caracteriza pela investigação a respeito das técnicas e das metodologias necessárias para o projeto, a elaboração e a gestão de websites para bibliotecas universitárias.

Com o objetivo de identificar as características específicas e necessárias para o trabalho com websites, procura circunscrever as etapas de implementação e implantação de uma página web específica para bibliotecas univeritárias. Assim, busca-se neste relato a proposição de uma metodologia experimental em três etapas fundamentais: projeto, elaboração e gestão, as quais descrevem os passos necessários para o trabalho com websites.

\section{PROJETO}

As etapas utilizadas durante o processo de construção de um website compreendem a tríade Projeto, Elaboração e Gestão. Sendo a primeira um momento de planejamento do website que se fundamenta em áreas do design de interfaces e de informação, materializando-se na concepção de projetos de navegação, identidade visual e arquitetura da informação.

O projeto de um website se expressa por meio da organização sistemática dos links para fornecer acesso à informação e criar associações com significado. Consiste na concepção dos percursos que podem ser usados pelo usuário no processo de busca pela informação. Sob esse aspecto, envolve design gráfico, tipografia, linguística, psicologia, ergonomia, computação e outras áreas relacionadas. Também envolve o projeto arquitetural da informação, foca-se na definiç̧ão da estrutura de hipermídia, na aplicação de padrões e na construção de modelos de interface. O objetivo é definir de que maneira a informação estará disposta para ser buscada no website e como os usuários poderão navegar nela (KALBACK, 2009). 
Essa fase da tríade pode ser proposta operacionalmente em duas etapas: especificações do projeto (foco na elaboração conceitual de possíveis componentes de navegação e interface juntamente com a apresentação de conteúdo) e implementação tecnológica e testes (foco na tecnologia que irá compor a estrutura dos projetos de design operacional e avaliativo). Na primeira etapa, concentram-se questões ligadas ao planejamento do projeto de design de navegação e de interface. Na segunda, define-se a estrutura de organização do conteúdo e as decisões relacionadas à escolha de ferramentas e tecnologias que irão proporcionar o desenvolvimento e a utilização do website.

Primeiramente, são definidos os conceitos sobre o perfil de usuários e suas demandas de informação, os objetivos do site, o design de navegação e a identidade visual do website.

O perfil de usuários de um website define-se primeiramente por meio da identificação de uma comunidade específica de utilizadores. Por exemplo, os discentes, os docentes e os técnicos que fazem uso de um acervo local e demais serviços de informação prestados por uma biblioteca universitária em seu ambiente físico. Esses usuários são potencialmente aqueles que irão utilizar o website da biblioteca.

Assim, torna-se fundamental a captura das características relevantes a esses usuários potenciais, o que inclui compreender suas experiências, seus conhecimentos e suas preferências por produtos de informação. A identificação dessas características, além de servir para identificação do perfil dos usuários do website, serve para auxiliar na tomada de decisão sobre o objetivo do site e a organização do conteúdo.

Muitas vezes, isso pode ser proposto operacionalmente por meio de um storyboard, ou seja, uma simulação simples do que virá a ser o website. Esse processo pode ser feito com papel e caneta ou utilizando-se ferramentas de modelagem para o layout do website, como os wireframes, ou seja, um esqueleto do sistema de navegação independente do design visual final que compõe uma camada primária de informações necessárias a cada página do website. Essas ferramentas visuais são usadas para descobrir se o layout oferecerá uma solução apropriada para o objetivo do website (KALBACK, 2009). 
Como exemplo, para esse tipo de software, sugere-se o Pencil Project's, ${ }^{2}$ ferramenta Open Source para criar rascunhos que irão dar a ideia do que um website ou aplicativo pode parecer. A finalidade de um wireframe criado com esse aplicativo é comunicar o layout das páginas sem ser preciso se ater a cores ou outros elementos de design nessa fase do projeto.

Outro ponto do projeto é o design de navegação, o qual consiste na concepção dos percursos que podem ser usados pelo usuário no processo de busca pela informação no website. O seu objetivo é dar a impressão ao usuário de que ele pode escolher livremente o seu percurso pela aplicação. Segundo Kalback (2009, p. 195), “[...] trata de coordenar objetivos, conteúdo, tecnologia e as necessidades dos usuários em uma experiência de navegação coesa." Com base nesse conceito é que se planeja a sistematização da interface de um website.

A sistematização das informações define os modos de recuperação da informação em cada um dos esboços gráficos, de cada ecrã que detalha a esquematização da navegação principal do conteúdo do website, especificando a interligação e a localização precisa dos elementos interativos que permitirão a navegação entre os assuntos abordados para ilustrar o objetivo do site e a sua interface de busca e recuperação de informações. Esse esquema pode ser representado visualmente, conforme a figura 1:

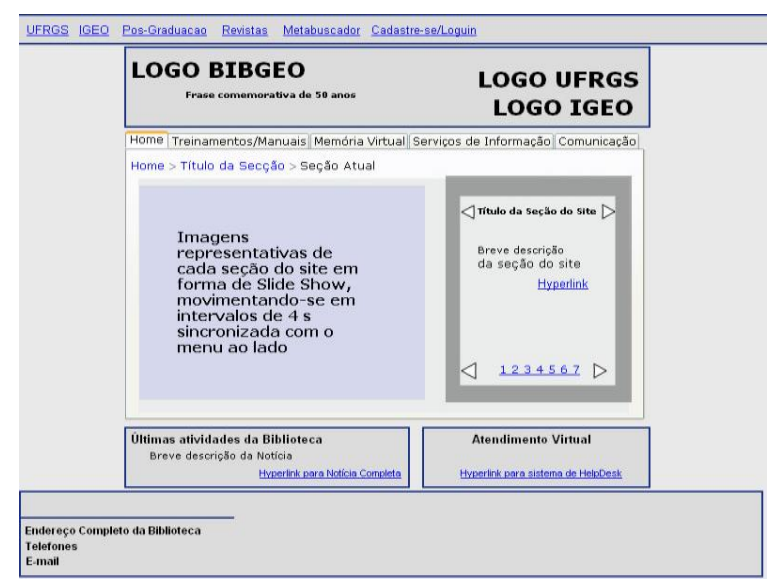

Figura 1 - Principal wireframe de um website

\footnotetext{
${ }^{2}$ Plugin gratuito para o navegador web Firefox, entre suas características incluem-se built-in stencils, suporte a documentos multipágina e múltiplas opções de exportação (HTML, PDF, PNG, JPG). Disponível em: http://pencil.evolus.vn/en-US/Home.aspx Acesso em: 23 de maio 2012
}

${ }^{3}$ Fonte: $\mathrm{O}$ autor

\begin{tabular}{l|l|l|l|l|l|l}
\hline (C) Rev. digit. bibliotecon. cienc. inf. & Campinas, SP & v.11 & n.1 & p.72-82 & jan./abr. 2013 & ISSN 1678-765X \\
\hline
\end{tabular}


A interface do usuário final deverá resultar na combinação de conteúdos e estrutura de apresentação. Deve ilustrar as possibilidades de interação entre usuário e a aplicação, proporcionando que ele possa navegar pelas seções de conteúdo planejadas no wireframe.

O passo seguinte indica o processo de desenvolvimento da interface do website, por meio da aplicação de técnicas e ferramentas de design visual. A partir de agora, é preciso preocupar-se com as imagens, as cores e a tipografia que irão compor a proposta visual.

O planejamento do design visual ${ }^{4}$ do website explora as características de concepção e desenho da interface. É possível comparar esse processo ao trabalho de arquitetos, engenheiros e artistas quando desejam construir algo; aqui expressamos e materializamos o website enquanto interface de comunicação mediada entre a biblioteca e os seus usuários.

O projeto de identidade visual define o design visual como uma estratégia de organização e apresentação do conteúdo e dos aspectos relacionados à aparência estética da interface. Esses elementos indicam o design da informação que irá compor a interface visual, ou seja, as diferentes formas de se mostrarem informações de forma clara e compreensiva.

Nesse contexto, pode-se citar a escolha da cor de um website, por exemplo o azul. Essa cor é considerada uma tonalidade fria, representa uma gama de qualidades abstratas. Como explica Swann (1993, p. 41, tradução nossa), o azul “[...] busca passar a ideia de integridade e estabilidade para proporcionar a sensação de confiança." Pode-se dizer que a cor azul será um dos fatores de maior relevância aos elementos que constituem a identidade visual do website, como: imagens de fundo, logotipos, fontes e outros signos gráficos, já que antes mesmo de se iniciar a leitura textual em um website lê-se a mensagem produzida por suas cores.

Definidas as questões ligadas ao projeto, como identificação de um perfil de usuários; objetivos do site; design de navegação; e identidade visual do website, o fluxo de trabalho remete para a próxima etapa metodológica, a Elaboração.

\footnotetext{
${ }^{4}$ Neste contexto do projeto, o termo design aparece como definidor dos processos de concepção de um modelo de interface e interação do website.
} 


\section{ELABORAÇÃO}

A elaboração resulta no desenvolvimento tecnológico do website com base nos projetos práticos realizados na primeira etapa da tríade. Esta etapa se caracteriza pela implantação e pela customização de sistemas e pelo uso de ferramentas tecnológicas e linguagens de programação.

Uma das primeiras questões que surgem no processo de desenvolvimento de um website é: desenvolver o projeto a partir do zero ou programar por meio de um Content Management Systems. Um CMS é um sistema geralmente implementado como um aplicativo web utilizado para facilitar o gerenciamento de conteúdo e documentos na web. Usados para gerenciar e controlar de maneira dinâmica o conteúdo e navegação de websites, os CMSs também permitem a geração de conteúdo interativo como fóruns, $\log s$, galerias de fotos e vídeos, sistemas de notícias entre outras aplicações específicas (MEHTA, 2009).

Ambas as maneiras possuem vantagens e desvantagens. Desenvolver um website a partir do zero certamente pode aumentar o tempo de desenvolvimento e o retrabalho; porém, pode facilitar a criação de aplicações específicas e autorais.

Por sua vez, o uso de um CMS facilita e agiliza a gestão de conteúdo no sentido de que exige menos conhecimentos aprofundados em linguagens de programação, além de facilitar a gestão de recursos básicos ligados a manutenção, atualização e administração do website. No entanto, é importante ressaltar que é preciso ter conhecimentos intermediários para utilizar esse tipo de sistema com eficácia (WELLING; THOMSON, 2006).

Os sistemas de gerenciamento de conteúdo facilitam a criação, a distribuição, a publicação e a disponibilidade de informações na internet. Os CMS são ferramentas criadas para facilitar o oneroso trabalho de editar e gerenciar as páginas de um website (TAROUCO et al., 2009)

Devido à capacidade desses sistemas de facilitarem o processo de gerenciamento de conteúdo, sugere-se neste estudo que aquele que iniciar a implementação de um website de biblioteca universitária utilize um CMS.

Como explica Kalback (2009, p. 205), esse tipo de sistema possibilita o “[...] armazenamento e recuperação do conteúdo e gerência o fluxo de trabalho para criar e 
manter o conteúdo em um website." Um CMS gerencia a informação digital no decorrer de seu ciclo de vida na web. Como exemplo de um CMS que pode ser utilizado por bibliotecas universitárias, sugere-se o Joomla.

Esse software foi criado em 2005 a partir de outro CMS, chamado Mambo. O Joomla integra as ferramentas necessárias para criar, gerir (editar e inserir) sem a necessidade de programação de código-fonte. Possui uma comunidade de desenvolvedores atuantes, o que faz com que exista uma serie de módulos, plugins e componentes disponíveis para adaptação.

Essa fase também se preocupa com a proposição de serviços de informação e com a análise do uso do website. No que tange à elaboração de serviços de informação, o desenvolvimento constante das tecnologias de comunicação e informação exige novas práticas para a organização e o tratamento de informação digital. Algumas delas materializam-se em aplicações web como o Mashup, ${ }^{5}$ que possibilita a implementação de conteúdo dinâmico para websites por meio de recursos disponíveis em outros locais da web. Uma aplicação web que recupera informações de fontes de dados externos.

O Mashup permite agregar conteúdo dinâmico (extraído de bases de dados em formatos distintos, combinados com outras informações). A combinação de dados de fontes distintas acontece, muitas vezes, com o uso de linguagens de marcação e serviços de servidor web como o XML e os XML web services.

Conforme o esquema exposto na figura 2, é possível visualizar a arquitetura de uma aplicação web que use Mashup:

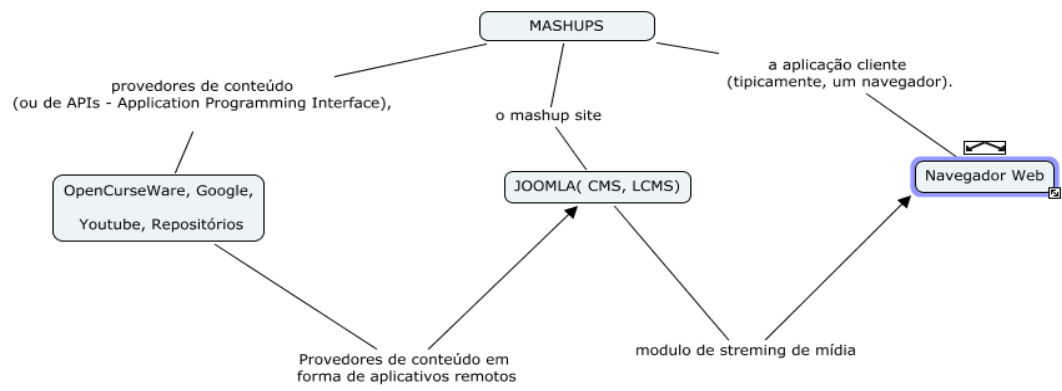

Figura 2 - Arquitetura Web Mashup ${ }^{6}$

\footnotetext{
${ }^{5} O$ termo Mashup se refere às interfaces e às aplicações web que combinam conteúdo de diferentes lugares em algo novo. Por Mashup se entende o novo gênero de aplicações web que possibilitam a reutilização de conteúdo de um site por outro, por exemplo, os mapas do Googlemaps.

${ }^{6}$ Fonte: $\mathrm{O}$ autor
} 
A arquitetura de uma aplicação Mashup é constituída pelos seguintes elementos, separados físico e logicamente: provedores de Application Programming Interface (API) de conteúdo, o sítio web que recebe o conteúdo e o navegador web do cliente, ou seja, o usuário final do conteúdo. A primeira camada, os provedores de conteúdo, consolida-se por meio de serviços web acessados via APIs de conteúdo. A segunda camada é o Mashup site - uma aplicação, web cliente do provedor de conteúdo, que atua como intermediária disponibilizando o conteúdo ao usuário final. É o lugar onde o Mashup está hospedado, onde ele é executado. (MERRILL, 2006)

Segundo Darlene Fichter, bibliotecária canadense da Universidade de Saskathewan, os Mashups têm proliferado na web por duas razões: recentemente, grandes companhias de internet, como Google e Yahoo, abriram seus dados para serem usados por outras fontes sem grandes complicações de licenças. A outra razão está ligada ao rápido crescimento das ferramentas para criação de um Mashup, sendo as mesmas de uso fácil e não exigindo know-how de programação avançados, ou seja, são simples de criar e exigem pouco conhecimento técnico (FICHTER, 2010).

Definida a estrutura tecnológica, surgem preocupações com a análise da informação que compõe o website, ou seja, com a gestão.

\section{GESTÃO}

A gestão preocupa-se com o processo de gerenciamento e análise da informação digital, ou seja, mede e avalia os fluxos da informação e da comunicação no website. Assim, considera-se a aplicação de métodos webométricos no processo de gerenciamento de conteúdo e elaboração da estrutura de websites. Os estudos webométricos podem ser de grande valia para projetistas e desenvolvedores de aplicações web. Nesse sentido, o interesse por esse campo de estudos fundamenta e justifica a abordagem aqui proposta. Acredita-se que a sistematização de informações em ambiente web defina novos modos de organização e recuperação da informação (VANTI, 2010).

O conceito de webometria permite a elaboração de mapas cognitivos e modelos matemáticos do website, o que facilita a compreensão de seu funcionamento, conteúdo e estrutura. 
Por meio de componentes de software e ferramentas estatísticas, é possível preparar o website para que ele seja focado em atender a determinados indicadores web. Esses tipos de indicadores, segundo Vanti (2010, p. 189), “[...] constituem valioso subsídio para avaliação das atividades desenvolvidas por diferentes instituições no espaço da internet." A mesma autora os classifica em três categorias: descritivos (contabilizam o tamanho ou o número de objetos que um espaço web apresenta), de conectividade (têm por finalidade o exame das conexões entre sites, enfocando os links externos que um espaço recebe quanto aos links que o espaço aponta) e os de popularidade (a utilização da informação, mensurada por meio de números e de características das visitas ao website) (VANTI, 2010).

Nesse contexto, podemos fundamentar a etapa da gestão com a análise métrica da informação por meio da medição do uso do website. Em específico, com o estudo da informação em qualquer formato, por meio da combinação de técnicas de recuperação da informação e medição dos fluxos informacionais em bases de dados web.

\section{CONSIDERAÇÕES FINAIS}

Em suma, este estudo propôs uma metodologia experimental, composta pela tríade projeto, elaboração e gestão de websites para bibliotecas universitárias. Focalizou-se em compreender o trabalho sob o ponto de vista de um profissional bibliotecário preocupado com questões relacionadas à informação digital e ao desenvolvimento web. Imerso no universo das novas tecnologias digitais para a web, buscou questionar-se sobre o que é necessário para adquirir o know-how com esse tipo de prática.

Assim, a primeira etapa da tríade, consolida-se como a organização sistemática da informação que irá compor o website. O projeto se define com a identificação do perfil de usuários, suas demandas de informação e pela elaboração dos wireframes do website. Isso porque identificar os usuários é tarefa fundamental para a proposição dos objetivos do site, bem como, a elaboração de projetos de design de navegação e identidade visual são estratégias norteadoras para o que vai ser implementado por meio de ferramentas tecnológicas e linguagens de programação. 
Dessa forma, no que tange o contexto tecnológico, a escolha inicial da etapa de elaboração fundamenta-se na questão: usar um CMS ou começar do zero? Como resposta a essa questão, sugere-se que não se inicie o desenvolvimento do zero, mas se opte pelo uso de um CMS como o Joomla. Ainda assim, não se exclue que o processo de planejamento e elaboração da interface do website necessite do aprendizado de conceitos que fundamentam a prática e o uso de ferramentas tecnológicas, como softwares e linguagens de programação utilizados para o desenvolvimento do design de interfaces.

A revisão técnica e criativa faz surgirem preocupações com a gestão website, ou seja, tudo aquilo que foi projetado e depois elaborado por meio da tecnologia precisa ser gerenciado. A necessidade de realizar-se a análise métrica do conteúdo e do uso da informação que compõem o website, pois se acredita que isso irá garantir o processo de identificação de erros nas funcionalidades do website e apontar as necessidades e demandas de informação e conteúdo por parte dos utilizadores. Ainda, sob a perspectiva da gestão, propõe-se o uso da webometria como método norteador para o desenvolvimento web.

A gestão de uma website naturalmente deve se preocupar com procedimentos de atualização, migração e preservação de informação em meio digital, temáticas que extrapolam esse estudo, mas não podem deixar de ser lembradas, pois são fundamentais.

Por fim, conclui-se que a tríade projeto, elaboração e gestão fazem parte de um processo contínuo e circular. Em outras palavras, não são etapas lineares que se seguem, estas etapas são autônomas e dependentes entre si, é possível regressar ou avançar nas etapas se o objetivo for qualificar os projetos ou melhorar os aplicativos elaborados por meio da tecnologia. E isso porque o trabalho de elaborar um website é algo permanente, que exige a atualização constante a respeito das técnicas, das tecnologias e das metodologias de desenvolvimento web e informação em meio digital. 


\section{REFERÊNCIAS}

FICHTER, D. What is a Mashup? In: ENGARD, N. Library Mashups Exploring new ways to deliver library data. Information Today, 2010.

KALBACH, James. Design de navegação Web. Trad. de Eduardo Kessler Piveta. Porto Alegre: Bookman, 2009.

MEHTA, Nirav. Choosing an Open Source CMS: find the best CMS and start working with it to create web sites, blogs, communities, e-commerce sites, and intranets. Birmingham: Packt Publishing, 2009.

MERRILL, D. Mashups: The new breed of Web app. IBM, 2006. Disponível em: < http:// www.ibm.com/developerworks/web/library/x-mashups/index.html >. Acesso em: 23 de maio 2011.

SWANN, A. El color en el diseño grafico: principios y uso del color. Barcelona: Editorial Gustavo Gili, 1993.

TAROUCO, L. M. Multimídia Interativa: princípios e ferramentas. Renote - Revistas Novas Tecnologias em Educação. V.7, p.1-13, 2009.

VANTI, N. Mapeamento das instituições federais de ensino superior. Informação \& Informação, Londrina, v.15, n.1, 2010. p. 55-67. Disponível em:

<http://www.uel.br/revistas/uel/index.php/informacao/article/view/4704/5878>. Acesso em: 23 de maio 2012.

WELLING, Luke; THOMSON, Laura. PHP e MYSQL: desenvolvimento Web. Rio de Janeiro: Elsevier, 2006.

\section{Como citar este artigo:}

SEMELER, Alexandre Ribas. Concepção de interfaces para websites de bibliotecas universitárias: projeto, elaboração e gestão de informação em meio digital. Rev. digit. bibliotecon. cienc. inf., Campinas, SP, v.11, n.1, p.72-82, jan./abr. 2013. ISSN 1678-765X. Disponível em:

<http://www.sbu.unicamp.br/seer/ojs/índex.php>. 